\title{
Research Progress towards Fishing Vessel Technology for Sustainable Use of Fisheries Resources
}

\author{
NAOYA UMEDA ${ }^{1}$ \\ 1 Osaka University, Suita, Osaka 565-0871, Japan (umeda@naoe.eng.osaka-u.ac.jp)
}

SUMMARY: For realising sustainable use of fisheries resources, new types of fishing vessel system should be developed because existing system has been implicitly optimised for unlimited amount of fish resources. Several research attempts in this research direction of fishing vessel technology are overviewed.

In techno-economic analyses on fishing vessel operation, the author updated the procedure for dynamically modelling variation of fish biomass as well as technical aspects of fishing vessel and cash-flow. As a result, his numerical results provided both possible scenarios towards overfishing and sustainable utilisation.

For safety and environment protection, relationship between new international regulations and recent researches is remarked. Efforts for improving onboard working conditions, including riding comfort and automatic control, and environment prevention, including use of wind energy, are also mentioned.

\section{KEYWORDS: sustainable use, safety, environment, automatic control}

\section{INTRODUCTION}

Population explosions in developing countries, several diseases of farm animals in developed countries and so on might lead to drastic increase of demand of fisheries resources as food. On the other hand, Food and Agricultural Organization of the United Nations (FAO) presumes that the total fish catch of the world in 1989 was comparable to the Maximum Sustainable Yield (MSY) of fisheries resources of the Globe. This means that our current fishing ability is capable to result in overfishing on the earth. However, since fisheries resources are reproducible with appropriate control, sustainable use of them is still possible for supplying food. Therefore, the Ocean Law Convention of the United Nations came into force in 1994 requires each nation to execute appropriate management of fisheries resources of its own EEZ. For realizing such sustainable use of fisheries resources, both preservation of ecology system and profitability of fisheries industry are indispensable. Nevertheless, since the current fishing vessel system has not yet well been adjusted to the above concept, it often makes overfishing without sufficient profit and sometimes it results in loss of fishermen's lives at sea by accidents. Moreover, fishing operation may induce environmental issues on the earth. Thus it is urgent to develop new fishing vessel system optimised to the sustainable use of fisheries resources. This paper overviews several researches in such direction on techno-economic system evaluation, safety improvement, working conditions, environment protection and so on.

\section{TECHNO-ECONOMIC EVALUATION}

Toward the sustainable utilisation of natural resources, it is essential to predict effect of fishing effort on both biomass and profitability of fisheries industry. Traditionally this prediction has been performed by a regression analysis with accumulated statistics. ${ }^{1)}$ However, such predictions have often faced great difficulties because prompt advance of fishing-related technology and significant change of biomass quite easily make the accumulated statistics out of date. To overcome these difficulties a more analysis-oriented evaluation is expected. Fishing operation is related with fish population dynamics, marine ecology, naval architecture, marine engineering, acoustic engineering, fishing technology, economics and so on. Thus the evaluation procedure have to take 


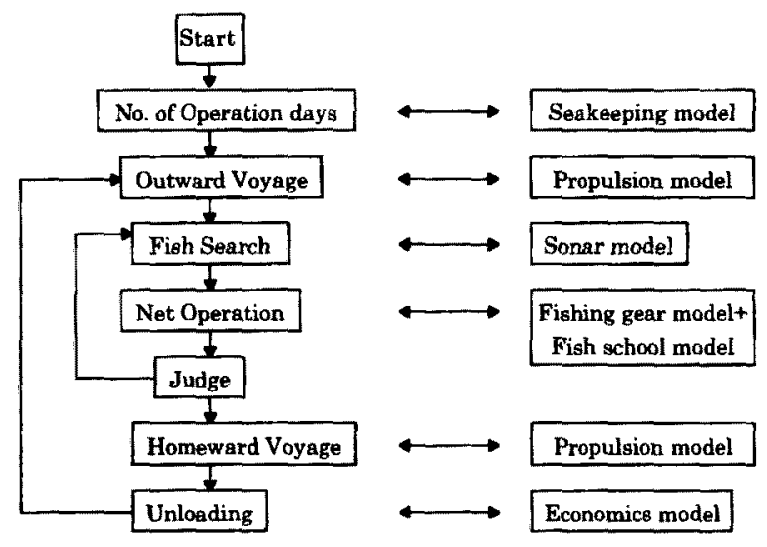

Fig.1 Overall procedure for techno-economic evaluation on purse seine fisheries

these aspects into account.

A pioneering model by Hamuro ${ }^{2)}$ considered some of the above elements for evaluating fisheries industry but did not directly consider fish population dynamics. As an extension of his model, the author ${ }^{3)}$ proposed a more comprehensive procedure, even with fish population dynamics taken into account, for a non-group purse seiner fleet. Numerical examples based on this new procedure showed effects of the fishing fleet size and provided both possible scenarios toward overfishing and the sustainable utilisation.

The techno-economic evaluation procedure applied to a non-group purse seiner fleet is summarised as follows. ${ }^{3)}$ The procedure quantitatively deals with the following whole sequence of fishing operation, as shown in Fig. 1.

The purse seiner leaves a fishing port and navigates toward a fishing ground by consuming fuel oil. This process is analysed with a propulsion model. Given a hull form, propeller and marine engine, the amount of fuel consumption and steaming hours are estimated by using a standard procedure of naval architecture.

As soon as she arrives on the fishing ground, she starts to search for fishing schools with a sonar. This process is analysed with a sonar model. Given an effective search radius of the scanning sonar, ship velocity and distribution of fish school, the averaged time duration for finding a fish school worthy to be shot is obtained by comparing searching area and total area of fishing ground.

If a fish school worthy to be caught is found,

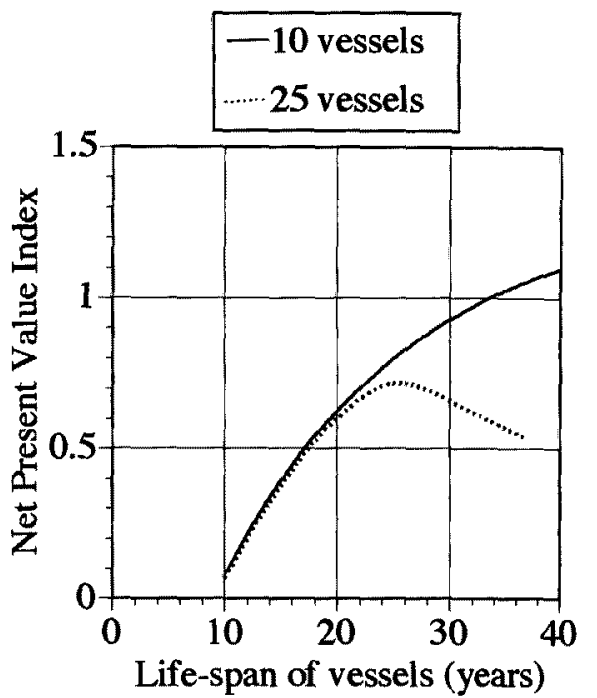

Fig. 2 Net present value index of the purse seiner fleet

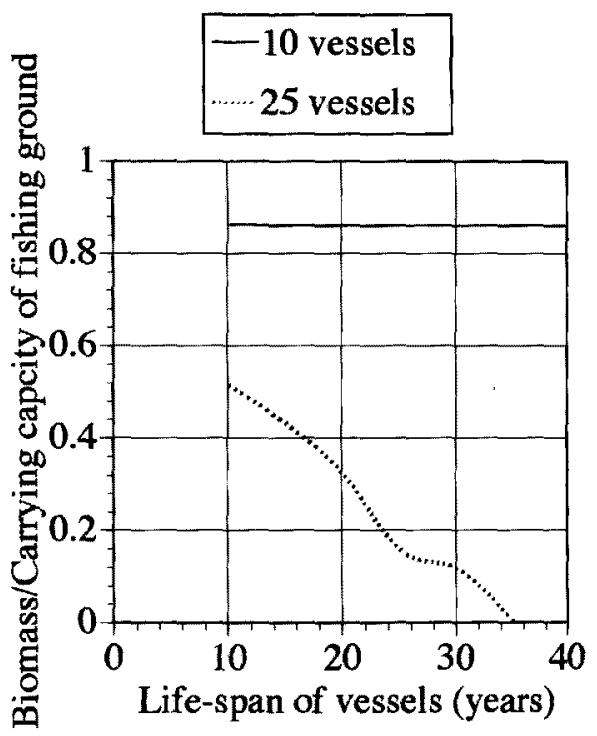

Fig. 3 Biomass with the purse seiner fleet operation

a purse seine is casted from the turning vessel. If the fish is screened by the net, they are loaded into fish hold of the vessel. As a result, fish biomass in the fish ground is changed. This process is analysed with a fishing gear model. Given a net size and distribution of fish school, the average catch per the one shot is estimated by comparing a screened volume and a fish school volume. Here the dynamic behaviour of biomass is modelled with the logistic model directly considering the effect of fish catch. 
If the vessel affords next shooting, she resumes to search for another fish school. If not, she leaves the fishing ground and returns to the fishing port. This judgement is realised also in the evaluation procedure.

When the vessel arrives at the port, the fish catch is unloaded ashore and sold at a market. The economic model estimates the earnings from fish catch and the cost covering the vessel, crew, gear, fuel oil, fresh water, repair, insurance, administration and so on.

The vessel is assumed to repeat above sequence during a year. However, sea state occasionally disturbs the above sequence. The seakeeping model estimates number of operation days by using wave statistics.

Numerical calculations based on the above procedure were carried out for purse seiner fleets of three different sizes; 50,25 and 10 vessels in a certain fishing ground. The vessel corresponds to the non-group purse seiner proposed by the Fishing Boat Association of Japan. ${ }^{5)}$ The fishing ground is primarily hypothetical but its framework is based on the Sardine one off South-eastern Hokkaido in 1980 's. ${ }^{6}$. The details were reported in the previous paper. ${ }^{3)}$ The results for the case of 50 vessels indicated that the target fish species became extinct without any profits and the first symptom of overfishing is observed in the searching hours and not in the amount of fish catch. The results for the case of 25 vessels indicated that the fleets are profitable but cannot sustain the biomass. The results for the case of 10 vessels were regarded as an example of sustainable use of fisheries resource because the biomass is attracted into a fixed point. It was note-worthy that no significant difference in profit exists between 25 vessels and 10 vessels. As far as these results showed, the fuel cost is not an important factor for improving the profit.

In the numerical study, the effect of life-span of vessels was investigated with the same system parameters. Here we assume for calculating the Net Present Value Index (NPVI) that the appropriate rate of interest is six percent and the remainder is ten percent of the original vessel price. Fig. 2 shows that in the case for 10 vessels the NPVI increases with increasing the life-span of vessels but in the case for 25 vessels the NPVI has a peak value at 25 years of life-span. Fig. 3 shows that in the case for 10 vessels that biomass is constant but in the case for 25 vessels the biomass becomes extinct within 37 years. Thus, the decrease of NPVI for 25 vessels with more than 25 years of life-span is presumed as the decrease of fish catch. These numerical examples demonstrate that the following fact. If the biomass is sustained, the longer use of vessels is a very effective measure to improve the profitability. However, if the biomass is not sustained, such measure is not appropriate. Although the work described here deals with only purse seiner fleet, it can be extended to trawler fleet and so on.

\section{SAFETY OF FISHING VESSELS}

Since fishing vessels operate far from their bases despite their smaller size, they often suffer sea disasters such as capsizing. Such disasters can not only cause catastrophic damages to finance of their fishing companies but also prevent fishing industries to attract sufficient labour forces. Therefore, safety of fishing vessels has been one of the essential elements of fishing vessels technology for many years, and several international safety criteria have been provided.

Based on the collaboration among FAO, International Labour Organization (ILO) and InterGovernmental Maritime Consultative Organization (IMCO), the Code of Safety for Fishermen and Fishing Vessels was adopted in 1974. This code aims to provide the minimum requirements for safety of operation and design of fishing vessels of 24 metres and over in length as a guide to national regulations. In 1977 the Torremolinos International Convention for the Safety of Fishing Vessels was adopted. This covers watertight integrity, stability, machinery and electrical installations, fire protection, protection of the crew, life saving appliance, emergency procedures, radiocommunications and navigation equipment of fishing vessels of 24 metres and over in length. However, Asian fishing nations did not ratify this convention because most of requirements are based on European fishing vessels and are often too stringent for Asian fishing vessels. It is obvious that a slender fishing vessel used in Asian region does not have enough space for safety equipment as beamy European vessels. This difference in hull form between two comes from that in management of fisheries resources. Thus, the Torremolinos Protocol of 1993 was adopted, and enables the administrations to determine different requirements 

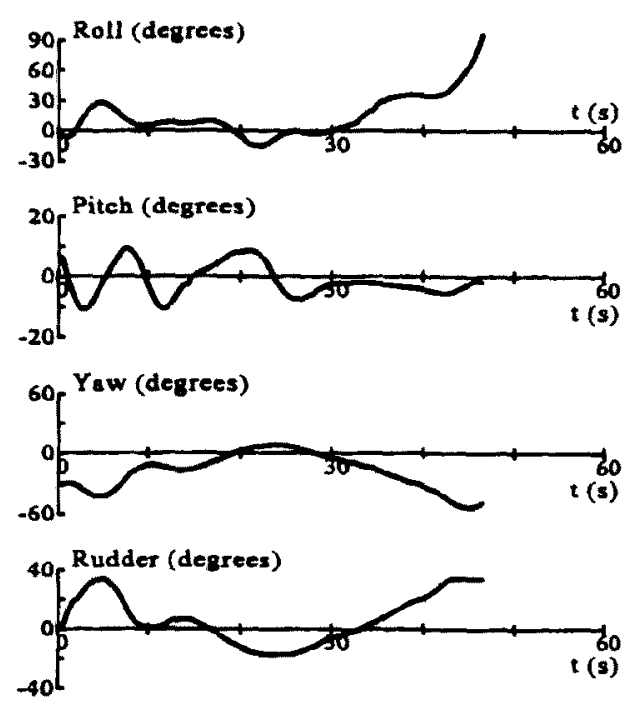

Fig. 4 Measured time series of capsizing due to broaching of a fishing vessel model complying with the IMO stability criteria. ${ }^{8)}$

on machinery and electrical installations, fire protection, life saving appliance and radiocommunications of fishing vessels of 24 metres and over but less than 45 metres in length as regional standards. Responding this revision, Asian nations adopted the guidelines of fishing vessels in the East and South-East Asia region by relaxing some requirements and European nations enforced their own regional standard as Council Directive $97 / 70 / E C$ without major relaxation. For fishing vessels of 12 metres and over but less than 24 metres in length, the FAO/ILO/ IMCO Voluntary Guidelines for Design, Construction and Equipment of Small Fishing Vessels was published in 1980. Although several standards exist, mandatory one has not yet been established and only the Fishing Vessels Safety Code and Voluntary Guidelines are internationally provided as recommendations. Currently revisions of these two are under discussion at International Maritime Organization (IMO).

As the stability requirement against capsizing, which induces fatal results, all the above standards utilise empirical criteria developed by Rahola. Applicability of Rahola's criteria has been criticized by many researchers. For example, capsizes of fishing vessels complying with these criteria in full or model scale, were reported as results of breaking waves or running in following and quartering seas. Dahle and Myrhaug ${ }^{6)}$ proposed a method for evaluating capsizing probability due to breaking waves. By using this method Iceland started a forecasting service of capsizing probability of fishing vessels through the Internet. ${ }^{7}$ For capsizes in following and quartering seas, systematic model experiments were carried out in a seakeeping and manoeuvring basins in Japan with several free-running ship models. ${ }^{8)}$ As a result, a procedure to determine critical metacentric height was established as well as the IMO Guidance to the master for avoiding dangerous situations in following and quartering seas (MSC/ Circ. 707). As the typical capsizing mode for a ship complying with Rahola's criteria, broaching has been identified, as shown in Fig. 4. This is a phenomenon that a ship cannot keep a constant heading angle despite of the maximum steering effort, and is explained as a kind of bifurcation phenomenon of a nonlinear dynamical system. ${ }^{9)}$ Recently the Society of Navel Architects and Marine Engineers (SNAME) established an Ad Hoc Panel on fishing vessels safety for discussing risk-based stability criteria and operational guidelines. $^{10)}$

\section{ONBOARD WORKING CONDITIONS}

Other than capsizing, working environment on fishing vessels operating at sea is too severe to gather younger labour force. As a result, fishing industry faces difficulties in keeping the sufficient number of crew and imparting skills to the next generation.

Ship motions in wave induce the inertia force on a fisherman on board. This can make his workability worse and sometimes make him fall out of the vessels. And the ship acceleration may result in seasickness of the fishermen. Nowadays ship motions, such as heave and pitch, can be calculated by a linear strip theory and the roll motions can be estimated by a nonlinear theory to some extent. Thus, by using these ship motion theories as tools researches to evaluate the workability and riding comfort are under way. ${ }^{11-12)}$ As a design for improving workability, fishing vessels with sheltered deck are recently increasing in northern Europe for preventing their crew from shipping waters.

Fishing operations such as midwater trawl require highly skilled fishermen, which cannot be easily found. To overcome such difficulty in 


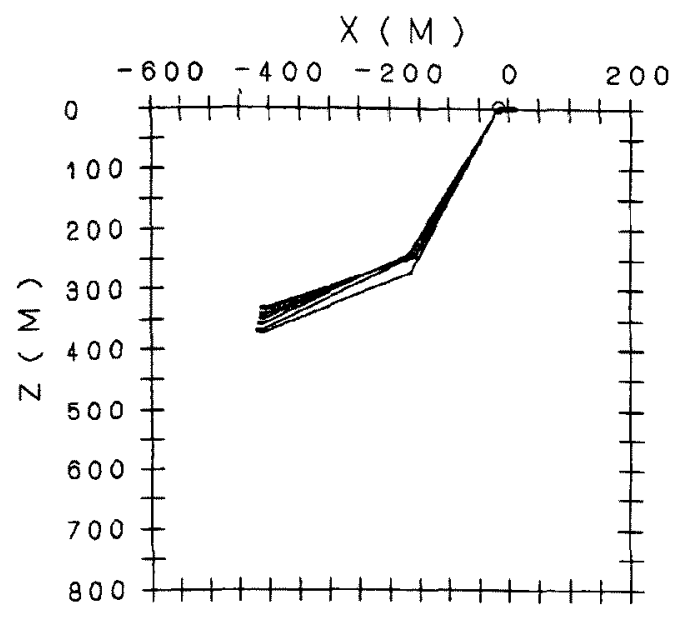

Fig. 5 Computer simulation of automatic control of a midwater trawl system. ${ }^{15)}$

midwater trawl, Hamuro ${ }^{13)}$ and then Muriaas ${ }^{14)}$ developed a control system, in which the manipulated variables are a propeller blade angle and a warp length and the controlled variables are net depth, net forward speed and so on. Later on Umeda ${ }^{15)}$ provided an automatic control law of this system using the optimal regulator theory and showed the possibility of automatic control, as shown in Fig. 5. Recently, combining such system with the GPS, the integrated trawl system, known as ITI, was developed for practical use. For longlining, automatic long-lining systems are widely used now. Automatic fishing operations will be one of the essential techniques for modern fishing vessels with help of control engineering, underwater acoustics and GPS.

\section{ENVIRONMENTAL ISSUES AND ENERGY SAVING}

Fishing industry like other industries has to be responsible for global environmental issues. Recently concern about effects of exhaust gas, involving $\mathrm{SO}_{\mathrm{x}}, \mathrm{NO}_{\mathrm{x}}, \mathrm{CO}_{2}$ and so on, from ships including fishing vessels on environment is spread. Thus, IMO adopted the new annex VI of the International Convention for the Prevention of Pollution from Ships, 1973, as modified by the Protocol of 1978, (MARPOL 73/78) in 1997. Here the upper limits of the sulphur content in fuel oil and the $\mathrm{NO}_{\mathrm{x}}$ emission from a diesel engine are specified. Responding these regulations, Hasegawa 16) carried out a research on a diesel engine of fishing vessels for reducing the $\mathrm{NO}_{\mathrm{x}}$ emission.
Moreover, the Kyoto Protocol to the United Nations Framework Convention on Climate may make IMO to require some reduction of $\mathrm{CO}_{2}$ emission from fishing vessels in future. For this purpose, energy saving of fishing operation is necessary. Thus hull form improvement of fishing vessels will be required further, and can contribute to also the profitability of fishing operation by reducing the fuel costs. Although such improvement was attempted with regression formula based on model test results in the past, computational fluid mechanics has been recently developed for practical application. Alternatively, the use of wind energy for fishing vessel propulsion can be a way to reduce the $\mathrm{CO}_{2}$ emission. In Japan a tuna long-liner with computercontrolled sails was built and used for commercial operations.

\section{CONCLUDING REMARKS}

In order to avoid 'food crisis' in the near future, it is urgent to develop new fishing vessels systems for sustainable use of fisheries resources. Although the research and development have already started as overviewed here, further research and exchange of ideas and experience are expected.

\section{REFERENCES}

1) Dahle E A. A Review of Quantitative Analysis of Vessels and Fishing Operations. Report of the Expert Consultation on Quantitative Analysis in Fishery Industries Development, 2, FAO, Rome, 1975: 1-24.

2) Hamuro C. Fishing Science. a part of the Handbook of World Fisheries (Fisheries Agency eds.), Norin Keizai Kenkyusyo, Tokyo, 1965: 719-736, in Japanese.

3) Umeda N. Techno-Economic Evaluation Procedure for a Non-Group Purse Seiner Fleet with Fish Population Dynamics Taken into Account. Fisheries Engineering, 1997; 33:175-184.

4) Report on Non-Group Purse Seine Fisheries. Fishing Boat Association of Japan, 1995:17-180, in Japanese.

5) Rahola J. The Judging of the Stability of Ships. Trans. INA, 1935.

6) Dahle E A. Myrhang D. Capsize Risk of Fishing Vessels. Schiffstechnik 1996; 43: 164-171.

7) Dahle E A, Myrhang D, Viggosson G. Information System on Waves and Stability of Small Fishing Vessels. 1997.

8) Umeda N, Matsuda A, et al. Stability Assessment for Intact Ships in the Light of Model Experiments. J Mar Sci Technol $1999 ; 4: 45-57$.

9) Umeda N. Nonlinear Dynamics of Ship Capsizing due to 
Broaching in Following and Quartering Seas. J Mar Sci Technol 1999; 4: 16-26.

10) Johnson B. On Developing a Rational and User-friendly Approach to Fishing Vessel Stability and Operational Guidance. Proceedings of the 5th International Workshop on Stability and Operational Safety of Ships, Trieste 2001.

11) Kimura N, Amagai K, Inaba $Y$. On the Relationship Between the Maintenance of Human Posture and the Ship Oscillatory Motions. Proceedings of World Symposium on Fishing Gear and Fishing Vessel Design, St. John's, 1988:564568.

12) Shigehiro R, Kuroda T, et al. Effect of Anti-Pitching Fins on Ship Motions from the Viewpoint of Passenger's Comfort. J Kansai Soc Nav Archit 2000;233:39-48, in Japanese.

13) Hamuro C. Development of Control Device for Midwater Trawl. Proceedings of National Conference on Fisheries Engineering Research, Choshi, 1991: 70-77, in Japanese.

14) Muriaas R. Automatic Trawl Control System. Proceedings of World Symposium on Fishing Gear and Fishing Vessel Design, St. John's, 1988:396-400.

15) Umeda N. Optimal Control of a Midwater Trawl System. Proceedings of the 4th Pacific Congress on Marine Science and Technology, Tokyo, 1990: 507-514.

16) Hasegawa $K$, Xue $Y$. Comparison of Combustion Characteristics between Emulsified Fuel and Low Cetane Number Fuel for a High Speed Diesel Engine. J Mar Eng Soc Japan 2000; 35:210-215, in Japanese. 